TAMKANG JOURNAL OF MATHEMATICS

Volume 33, Number 4, Winter 2002

\title{
ON THE CONVEX NULL OPERATORS
}

\section{JIAN-LIN LI}

\begin{abstract}
The object of this paper is to investigate some properties of the convex null operators which are defined for analytic functions. The result obtained here extends and sharpens some recent results due to Silvia [5], Ahuja and Jahangiri [1].
\end{abstract}

\section{Introduction}

A sequence $\left\{c_{n}\right\}_{n=0}^{\infty}$ of positive real numbers is said to be a normalized convex null sequence if $c_{0}=1,\left\{c_{n}\right\}_{n=0}^{\infty}$ is decreasing to 0 as $n \rightarrow \infty$, and $c_{n}-2 c_{n+1}+c_{n+2} \geq 0$ for each $n$. Let $M(2)$ denote the class of all such sequences (alternatively, such sequences are called monotonic of order 2. See [2]).

Let $A$ be the class of functions $f(z)$ of the form $f(z)=z+\sum_{n=2}^{\infty} a_{n} z^{n}$, which are analytic in the open unit disk $U=\{z: z \in C$ and $|z|<1\}$. For $\left\{c_{n}\right\}_{n=0}^{\infty} \in M(2)$, Silvia[5] defined the corresponding convex null operator $F_{\left\{c_{n}\right\}}$ on $A$ by $F_{\left\{c_{n}\right\}}(f)=z+$ $\sum_{n=2}^{\infty}\left(a_{n} / c_{n-1}\right) z^{n}$ for $f(z)=z+\sum_{n=2}^{\infty} a_{n} z^{n} \in A$. Corresponding to $\left\{c_{n}\right\}_{n=0}^{\infty} \in M(2)$ such that $F_{\left\{c_{n}\right\}}(z /(1-z)) \in A$, we use $F\left(\left\{c_{n}\right\} ; \beta\right)$ to denote the family of functions $f \in A$ such that $\operatorname{Re}\left(F_{\left\{c_{n}\right\}}(f)\right)^{\prime}>\beta$ in $U$. Recently, Silvia [5], Ahuja and Jahangiri [1] discussed some geometric properties of the class $F\left(\left\{c_{n}\right\} ; \beta\right)$. In the present paper, we first correct some erroneous results appeared in papers [5] and [1], we then extend and sharpen some results obtained by these authors.

\section{Preliminaries}

The following two lemmas play an important role in the proof of our improved results.

Lemma 1(Fejer [2]). If $\left\{c_{n}\right\}_{n=0}^{\infty} \in M(2)$, then the function $q(z)=c_{0} / 2+\sum_{n=1}^{\infty} c_{n} z^{n}$ is analytic in $U$ and $\operatorname{Re}\{q(z)\}>0$ in $U$.

Lemma 2. For $j=1,2$, let $H_{j}$ be analytic in $U, H_{j}(0)=1, \operatorname{Re}\left\{H_{1}(z)\right\}>\alpha$ and $\operatorname{Re}\left\{H_{2}(z)\right\}>\beta$ in $U$. Then

$$
\operatorname{Re}\left\{\left(H_{1} * H_{2}\right)(z)\right\}>2(\alpha+\beta)-2 \alpha \beta-1, \quad(z \in U)
$$

Received July 25, 2001.

2000 Mathematics Subject Classification. 30C55, 30C75

Key words and phrases. Convolution, convex null operator, differential subordination, extreme point theory. 
where "*" denotes the Hadamard product or convolution.

The conclusion of Lemma 2 readily follows from a well-know fact that if $P(z)$ is analytic in $U, P(0)=1$, and $\operatorname{Re}\{P(z)\}>1 / 2$ in $U$, then for any function $F(z)$, analytic in $U,(P * F)(U)$ is contained in the convex hull of $F(U)$.

\section{Theorems and Their Proofs}

(I) It is know that if $\left\{c_{n}\right\}_{n=0}^{\infty} \in M(2)$ then $\left\{c_{n} /(1+n)\right\}_{n=0}^{\infty} \in M(2)$. But we can't have $\left\{b_{n}\right\}_{n=0}^{\infty} \in M(2)$ for $b_{0}=1, b_{n}=c_{n} /(\gamma(1+n)) \quad(n=1,2, \ldots)$ and $1 / \gamma>0$. For example, let $c_{n}=(1+n)^{-1}$ and $\gamma=1 / 5$, we see that $b_{0}-b_{1}<0$ and $\left\{b_{n}\right\}_{n=0}^{\infty} \notin M(2)$. Therefore, Theorem 8 and the second part of Theorems 9 and 11 in [5] are not correct. In this section, we shall extend and sharpen the corresponding results to the following.

Theorem 1. If $f \in F\left(\left\{c_{n}\right\} ; \beta\right)$, then $\operatorname{Re}\{f(z) / z\}>2 \beta-1+2(1-\beta) \log 2$ in $U$.

Proof. For $f(z)=z+\sum_{n=2}^{\infty} a_{n} z^{n} \in F\left(\left\{c_{n}\right\} ; \beta\right)$, we write

$$
\frac{f(z)}{z}=\left(1+\sum_{n=2}^{\infty}\left(\frac{n}{c_{n-1}}\right) a_{n} z^{n-1}\right) *\left(1+\sum_{n=2}^{\infty} \frac{c_{n-1}}{n} z^{n-1}\right) .
$$

Since

$$
\operatorname{Re}\left\{1+\sum_{n=2}^{\infty} \frac{c_{n-1}}{n} z^{n-1}\right\}=\operatorname{Re}\left\{\int_{0}^{1} g(z t) d t\right\}
$$

for $g(z)=1+\sum_{n=1}^{\infty} c_{n} z^{n}$, we see from Lemma 1 that $\operatorname{Re}\{g(z t)\} \geq 1 /(1+t),(z \in U, t \in$ $[0,1])$, which yields

$$
\operatorname{Re}\left\{1+\sum_{n=2}^{\infty} \frac{c_{n-1}}{n} z^{n-1}\right\}>\int_{0}^{1} \frac{1}{1+t} d t=\log 2 \quad(z \in U) .
$$

From (1), (2) and Lemma 2, we get the desired result.

Theorem 2. If $f \in F\left(\left\{c_{n}\right\} ; \beta\right)$ for $\left\{c_{n}\right\}_{n=0}^{\infty}=\left\{(1+\alpha n)^{-1}\right\}_{n=0}^{\infty}, \alpha>0$ and $\beta<1$, then

$$
\begin{array}{ll}
\operatorname{Re}\left\{f^{\prime}(z)\right\}>\beta+(1-\beta)\left(2 \int_{0}^{1} \frac{d t}{1+t^{\alpha}}-1\right) & (z \in U), \\
\operatorname{Re}\left\{\frac{f(z)}{z}\right\}>1+2(\beta-1) \int_{0}^{1} \frac{t^{\frac{1}{\alpha}}-t}{(\alpha-1)(1+t)} d t & (z \in U) .
\end{array}
$$

These results are sharp. The extremal function is given by

$$
f_{1}(z)=z+\sum_{n=1}^{\infty} \frac{2(1-\beta)(-1)^{n}}{(1+n)(1+\alpha n)} z^{n+1}
$$


Proof. For $f(z)=z+\sum_{n=2}^{\infty} a_{n} z^{n} \in F\left(\left\{c_{n}\right\} ; \beta\right)$, we write

$$
f^{\prime}(z)=\left(1+\sum_{n=2}^{\infty}\left(\frac{n}{c_{n-1}}\right) a_{n} z^{n-1}\right) *\left(1+\sum_{n=2}^{\infty} c_{n-1} z^{n-1}\right)
$$

and $f(z) / z$ as $(1)$ for $c_{n}=1 /(1+\alpha n), n=0,1,2, \ldots$

Since

$$
\begin{aligned}
\operatorname{Re}\left\{1+\sum_{n=2}^{\infty} c_{n-1} z^{n-1}\right\} & =1+\operatorname{Re}\left\{\frac{1}{\alpha} \int_{0}^{1} t^{\frac{1}{\alpha}} \frac{z}{1-t z} d t\right\} \\
& >1+\frac{1}{\alpha} \int_{0}^{1} t^{\frac{1}{\alpha}} \frac{-1}{1+t} d t=1-\int_{0}^{1} \frac{t^{\alpha}}{1+t^{\alpha}} d t \quad(z \in U)
\end{aligned}
$$

we get (3) from (6) amd Lemma 2. Similarly, from

$$
\begin{aligned}
\operatorname{Re}\left\{1+\sum_{n=2}^{\infty} \frac{c_{n-1}}{n} z^{n-1}\right\} & =1+\operatorname{Re}\left\{\int_{0}^{1} \frac{t^{\frac{1}{\alpha}}-t}{\alpha-1} \cdot \frac{z}{1-t z} d t\right\} \\
& >1-\int_{0}^{1} \frac{t^{\frac{1}{\alpha}}-t}{(\alpha-1)(1+t)} d t \quad(z \in U)
\end{aligned}
$$

we obtain (4). The proof of Theorem 2 is complete.

Note that Theorem 2 sharpens the result [5, Theorem 9]. As noted by Silvia [5, Remarks 3 and 4], Ponnusamy [3] obtained (3) by using differential subordination, while extreme point theory was used by Silverman [4] to obtain Theorem 2 in the special case when $\alpha=1$.

The following Theorem 3 will correct and sharpen [5, Theorem 11].

Theorem 3. For $\alpha>0, \beta>0$, and $f \in A$, if

$$
\operatorname{Re}\left\{f^{\prime}(z)+(\alpha+\beta) z f^{\prime \prime}(z)+\alpha \beta z\left(z f^{\prime \prime}(z)\right)^{\prime}\right\}>0 \quad(z \in U)
$$

holds, then

$$
\begin{array}{ll}
\operatorname{Re}\left\{f^{\prime}(z)\right\}>1+2 \sum_{n=1}^{\infty} \frac{(-1)^{n}}{(1+\alpha n)(1+\beta n)} & (z \in U), \\
\operatorname{Re}\left\{\frac{f(z)}{z}\right\}>1+4 \log \frac{e}{2} \cdot \sum_{n=1}^{\infty} \frac{(-1)^{n}}{(1+\alpha n)(1+\beta n)} & (z \in U) .
\end{array}
$$

These results are sharp. The extremal function is given by.

$$
f_{2}(z)=z+2 \sum_{n=1}^{\infty} \frac{(-1)^{n}}{(1+n)(1+\alpha n)(1+\beta n)} z^{n+1} .
$$


Proof. Observe that for $\alpha>0, \beta>0$ and $f(z)=z+\sum_{n=2}^{\infty} a_{n} z^{n} \in A$, we have

$$
\begin{aligned}
& f^{\prime}(z)+(\alpha+\beta) z f^{\prime \prime}(z)+\alpha \beta z\left(z f^{\prime \prime}(z)\right)^{\prime}=1+\sum_{n=2}^{\infty} \frac{n a_{n}}{c_{n-1}} z^{n-1}, \\
& f^{\prime}(z)=\left(1+\sum_{n=2}^{\infty} \frac{n a_{n}}{c_{n-1}} z^{n-1}\right) *\left(1+\sum_{n=2}^{\infty} c_{n-1} z^{n-1}\right),
\end{aligned}
$$

where $c_{n}=(1+\alpha n)^{-1}(1+\beta n)^{-1}, n=1,2, \ldots$

Let $p(z)=1 / 2+\sum_{n=1}^{\infty} c_{n} z^{n}$. Then

$$
p(z)=\frac{1}{2}+\int_{0}^{1} \frac{t^{\frac{1}{\alpha}}-t^{\frac{1}{\beta}}}{\alpha-\beta} \cdot \frac{z}{1-t z} d t
$$

which yields

$$
\begin{aligned}
\operatorname{Re}\{p(z)\} & =\frac{1}{2}+\int_{0}^{1} \frac{t^{\frac{1}{\alpha}}-t^{\frac{1}{\beta}}}{\alpha-\beta} \cdot \operatorname{Re}\left\{\frac{z}{1-t z}\right\} d t \\
& >\frac{1}{2}+\int_{0}^{1} \frac{t^{\frac{1}{\alpha}}-t^{\frac{1}{\beta}}}{\alpha-\beta} \cdot \frac{-1}{1+t} d t=p(-1)>0 \quad(z \in U)
\end{aligned}
$$

Hence,

$$
\operatorname{Re}\left\{1+\sum_{n=2}^{\infty} c_{n-1} z^{n-1}\right\}>1+\sum_{n=1}^{\infty} \frac{(-1)^{n}}{(1+\alpha n)(1+\beta n)} \quad(z \in U) .
$$

By applying (12), (13), (16) and Lemma 2, we get the sharp result (9).

Since $\frac{f(z)}{z}=f^{\prime}(z) * p(z)$ for $p(z)=1+\sum_{n=2}^{\infty} \frac{1}{n} z^{n-1}$, and

$$
\operatorname{Re}\{p(z)\}=\operatorname{Re}\left\{1+\int_{0}^{1} \frac{t z}{1-t z} d t\right\}>\log 2
$$

in $U$, we obtain (10) from (9) and Lemma 2. The sharpness of Theorem 3 follows by considering the extremal function (11) directly. This completes the proof.

It should be point out that the above proof also shows that, for $\alpha>0, \beta>0$ and $f \in A$, the best bound $\sigma$ such that

$$
\operatorname{Re}\left\{f^{\prime}(z)+(\alpha+\beta) z f^{\prime \prime}(z)+\alpha \beta z\left(z f^{\prime \prime}(z)\right)^{\prime}\right\}>\sigma \Longrightarrow \operatorname{Re}\left\{f^{\prime}(z)\right\}>0 \quad(z \in U)
$$

is

$$
\sigma(\alpha, \beta)=1+\left[2 \sum_{n=1}^{\infty} \frac{(-1)^{n}}{(1+\alpha n)(1+\beta n)}\right]^{-1}
$$

The extremal function is given by

$$
f_{3}(z)=z+2(1-\sigma(\alpha, \beta)) \sum_{n=1}^{\infty} \frac{(-1)^{n}}{(1+n)(1+\alpha n)(1+\beta n)} z^{n+1} .
$$


(II) Ahuja and Jahangiri [1] obtained some results for the class $M_{n}(\alpha)=\{f(z) \in A$ : $\left.\operatorname{Re}\left(D^{n} f(z)\right)^{\prime}>\alpha, z \in U\right\}$, where $D^{n} f(z)$ is the Hadamard product of $f$ with $z /(1-z)^{n+1}$. It is easily seen that this class is equal to the class $F\left(\left\{c_{k}\right\} ; \alpha\right)$ with $\left\{c_{k}\right\}_{k=0}^{\infty} \in M(2)$ defined by

$$
c_{0}=1, \quad c_{k}=\left(\left(\begin{array}{c}
k+n \\
n
\end{array}\right)\right)^{-1}, \quad k=1,2, \ldots ; n \in N_{0}, \alpha<1 .
$$

We note with careful calculation that Theorems 4 and 5 of [1] are not correct. In fact, applying lemma 2 directly, we should have the following.

Theorem 4. Let $f \in M_{n}(\alpha)$ and let $g \in A$ so that $\operatorname{Re}\{g(z) / z\}>(n+\alpha) /(n+1)$ in $U$. Then $f * g \in M_{n}(\beta)$, where

$$
\beta=\frac{4 \alpha-2 \alpha^{2}+n-1}{n+1} \geq \alpha
$$

Proof. For $f(z)=z+\sum_{n=2}^{\infty} a_{n} z^{n} \in M_{n}(\alpha)$, we have

$$
\operatorname{Re}\left\{1+\sum_{k=2}^{\infty} k\left(\begin{array}{c}
k+n-1 \\
n
\end{array}\right) a_{k} z^{k-1}\right\}>\alpha \quad(z \in U) .
$$

Combining (21) with $\operatorname{Re}\{g(z) / z\}>(n+\alpha) /(n+1)$, we obtain from Lemma 2 that

$$
\operatorname{Re}\left\{\left(D^{n} f\right)^{\prime} * \frac{g(z)}{z}\right\}>\frac{4 \alpha-2 \alpha^{2}+n-1}{n+1}=: \beta \quad(z \in U) .
$$

This shows that $f * g \in M_{n}(\beta)$.

As it is shown in [1] that $g \in M_{n}(\alpha)$ implies $\operatorname{Re}\{g(z) / z\}>(n+\alpha) /(n+1)$ in $U$, we also have the following.

Theorem 5. Let $f$ and $g$ be in $M_{n}(\alpha)$. Then $f * g \in M_{n}(\beta) \subset M_{n}(\alpha)$, where $\beta$ is given by (20).

\section{References}

[1] O. P. Ahuja and M. Jahangiri, On the derivatives of a family of analytic functions, Math. Japon. 47(1) (1998), 67-72.

[2] L. Fejer, Uber die positivat von summen, die nach trigonometrischen order Legendreschen funktionen fortschreiter, Acta Litt. Ac. Sci. Szeged, 1925,75-86.

[3] S. Ponnusamy, Differential subordination and Bazilevic functions, Proc. Ind. Acad. Sci. Math. Sci. 105(2) (1995), 169-186.

[4] H. Silverman, A class of bounded starlike functions, Internat. J. Math. and Math. Sci. $\mathbf{1 7}(2)$ (1994), 249-252.

[5] E. M. Silvia, Convex null operators, Math. Japon. 47(2) (1998) 311-317.

Research Center for Science, Xi'an Jiaotong University, Xi'an, Shaan Xi 710049, P. R. China.

Department of Applied Mathematics, Northwestern Polytechnical University, Xi'an, Shaan Xi 710072, P. R. China (Permanent address). 